\title{
Numerical Study of Drift-Kinetic Evolution of Collisional Plasmas in Tori
}

\author{
C. O. Beasley, Jr. \\ H. K. Meier \\ W. I. van Rij \\ J. E. McCune
}




\section{DISCLAIMER}

This report was prepared as an account of work sponsored by an agency of the United States Government. Neither the United States Government nor any agency Thereof, nor any of their employees, makes any warranty, express or implied, or assumes any legal liability or responsibility for the accuracy, completeness, or usefulness of any information, apparatus, product, or process disclosed, or represents that its use would not infringe privately owned rights. Reference herein to any specific commercial product, process, or service by trade name, trademark, manufacturer, or otherwise does not necessarily constitute or imply its endorsement, recommendation, or favoring by the United States Government or any agency thereof. The views and opinions of authors expressed herein do not necessarily state or reflect those of the United States Government or any agency thereof. 


\section{DISCLAIMER}

Portions of this document may be illegible in electronic image products. Images are produced from the best available original document. 
Printed in the United States of America. Available from National Technical Information Service

U.S. Department of Commerce

5285 Port Royal Road, Springfield, Virginia 22161

Price: Printed Copy $\$ 4.50$; Microfiche $\$ 2.25$

This report was prepared as an account of work sponsored by the United States Government. Neither the United States nor the Energy Research and Development Administration/United States Nuclear Regulatory Commission, nor any of their employees, nor any of their contractors, subcontractors, or their employees, makes any warranty, express or implied, or assumes any legal liability or responsibility for the accuracy, completeness or usefulness of any information, apparatus, product or process disclosed, or represents that its use would not infringe privately owned rights. 
Contract No. W-7405-eng-26

\section{NUMERICAL STUDY OF DRIFT-KINETIC EVOLUTION \\ OF COLLISIONAL PLASMAS IN TORI}

C. O. Beasley, Jr., H. K. Meier, W. I. van Rij

Oak Ridge National Laboratory

and

J. E. McCune

Massachusetts Institute of Technology

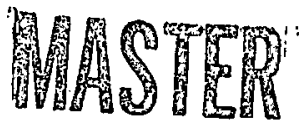

THERMONUCLEAR DIVISION

MARCH 1976

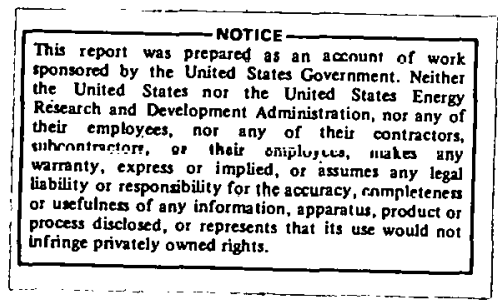

NOTICE This document contains information of a preliminary nature and was prepared primarily for internal use at the Oak Ridge National Laboratory. It is subject to revision or correction and therefore does not represent a final report.

OAK RIDGE NATIONAL LABORATORY

Oak Ridge, Tennessee 37830

operated by

UNION CARBIDE CORPORATION

for the

ENERGY RESEARCH AND DEVELOPMENT ALMINISTRATION 


\section{THIS PAGE}

\section{WAS INTENTIONALLY \\ LEFT BLANK}




\section{TABLE OF CONTENTS}

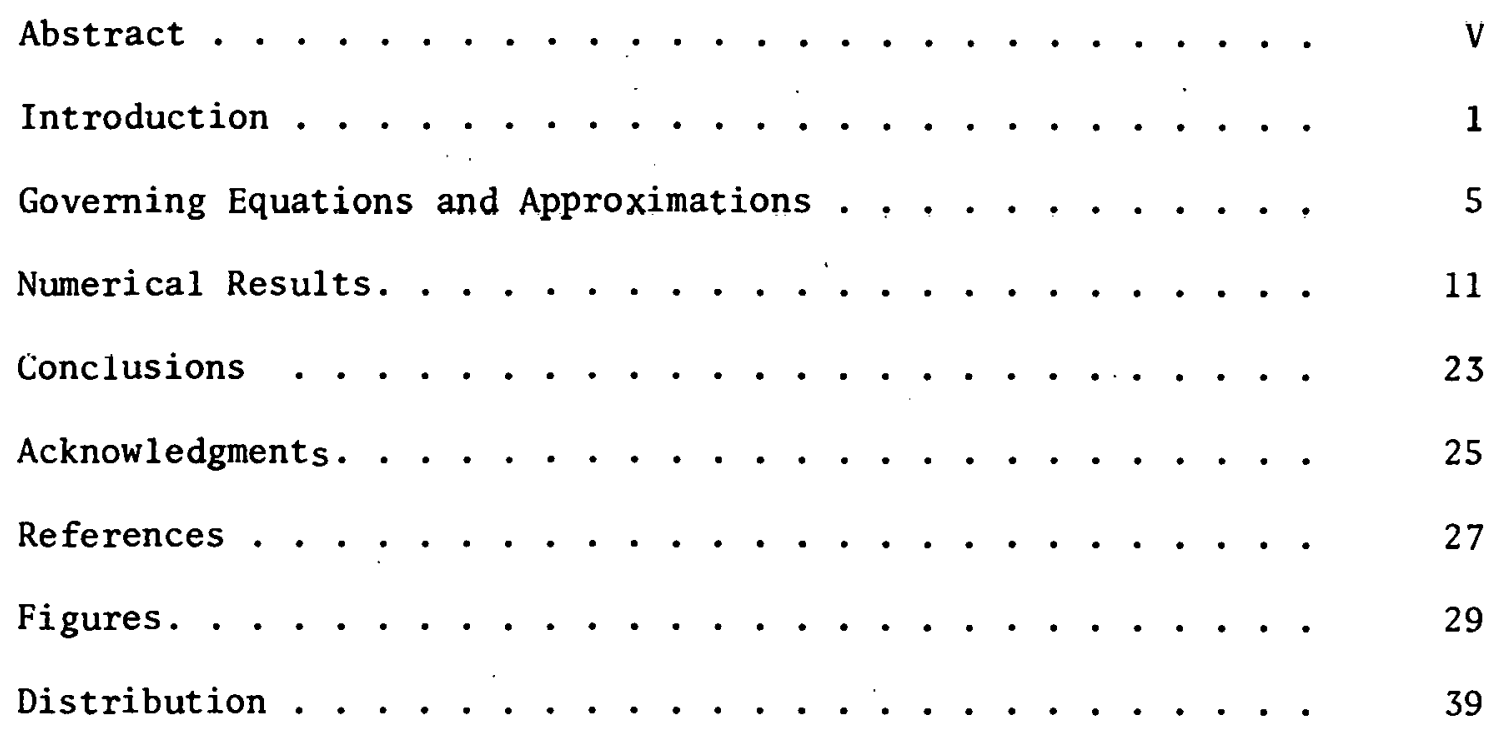


THIS PAGE

WAS INTENTIONALLY

LEFT BLANK 


\section{NUMERICAL STUDY OF DRIFT-KINETIC EVOLUTION \\ OF COLLISIONAL PLASMAS IN TORI*}

by

C. 0. Beasley, Jr., J. E. McCune, ${ }^{+}$H. K. Meier, and W. I. van Rij Oak Ridge National Laboratory, Oak Ridge, Tennessee

\section{ABSTRACT}

Preliminary numerical results for the dynamics of toroidally confined plasmas in the drift-kinetic, Fokker-Planck description are discussed. These solutions were obtained by using the techniques inherent to the collisional plasma model (CPM) - described in detail elsewhere. An initial value problem is solved in the local approximation in which collisions and particle dynamics compete in a given magnetic field to set up a quasi-equilibrium. Both the plasma (guiding center) distribution function and many macroscopic quantities of interest are monitored. Good agreement with corresponding but more approximate theories is obtained over a wide range of collisionality, particularly with regard to the neoclassical particle flux. Encouraging confirmation of earlier results for the distribution function is achieved when due account is taken of the differing collisionality of particles with differing energies. These initial results indicate the potential importance of certain non-local effects as well as inclusion of self-consistency between fields and plasma currents and densitics.

\footnotetext{
ॠResearch sponsored by the Energy Research and Development Administration under contract with Union Carbide Corporation.

${ }^{+}$Massachusetts Institute of Technology, Cambridge, Massachusetts
} 


\section{I . INTRODUCTION}

Previous calculations of neoclassical equilibrium and transport ${ }^{1-9}$ made use of analytic solutions of a drift-kinetic equation in which the effect of collisions was included. To make analytic solution possible, model collision operators of varying complexity (see Refs. 1 , 5,8 and 9 ) were assumed. In the early works, ${ }^{1-7}$. the solutions obtained were valid over limited ranges of collisionality. In later calculations $^{8,9}$ attempts were made to use models which permitted more general solutions; that is, these treatments were constructed to maintain validity over the entire range of collisionality. However, for this purpose, approximations were necessarily introduced which precluded the full description of the distribution function in all regimes and over all particle energies. ${ }^{9}$ In the interest of achieving more completeness and accuracy, use of numerical simulation has recently been introduced. 10

The purpose of our present work is to use a newly developed numerical method which offers a distinct alternative to the above-mentioned numerical simulation. For convenience, this technique is referred to here as the collisional plasma model (CPM). The CPM enables one to study the neoclassical equilibrium with no approximations within the driftkinetic ordering. Certain special features of the method used to generate the results given here are described in detail in Refs. 11 and 12. For the sake of completeness, a few of these features are also reviewed in Section II of this paper. 
In the present work, we begin our: calculations as an initial value problem in which the initial state of the plasma is described by a homogeneous, stationary Maxwellian distribution for each species, with imposed radial density gradients, a specified (model) magnetic field, and a given induction ring voltage. An initial temperature is specified, but heating of the plasma is then monitored, and the temperature varies with the amount of applied voltage and the degree of collisionality. Even though the time-dependent problem has not been treated self-consistently in this work (in that, for example, the poloidal magnetic field $B_{\theta}$ and the induction electric field $E_{\zeta}$ are imposed and taken to be time-1ndependent), the initial-value calculations themselves yield some interesting results. These will be discussed in the light of earlier initial-value MHD calculations (see Section III.A).

At the present state of development of the theory, the time evolution problem is used primarily as a means of allowing our numerical model of a toroidal plasma discharge to evolve until a quasi-steady state, as defined in Section III.B, is attaincd. The results of such equilibrium calculations show substantial and encouraging agreement with earlier results; ${ }^{6,8,9}$ these results will also be discussed (see Section III.B).

Although more general than those of earlier work, the results presented here remain subject to limitations in applicability, primarily because two approximations have been 1mposed for the sake of convenience in the initial studies. The first restriction is that the ion kinetics has not been included; the ions are simply treated here as a massive neutralizing background. Therefore, physical observables (heal flux, pressure, etc.) are not necessarily completely characteristic of tokamak 
observables. The second approximation is that of radial localization. The validity and limitations of this approximation are discussed in Sections II and III.A.

In Section II the basic equations are discussed briefly and the underlying physical assumptions and their implications are described. In Section III.A we discuss the results of the time-dependent numerical calculations and attempt to relate these to various physical phenomena as well as to other initial-value calculations. In Section III.B we show results of equilibrium calculations. Results are given for cases with radial density gradient alone, with temperature and density gradients both included, and with various values of the induction voltage. These results are obtained over a wide range of collisionality: $0.1 \leq \nu_{*} \leq 100$, where $\nu_{*}$ is defined as $\nu_{\text {eff }} / \omega_{\text {be }} ; \nu_{\text {eff }}$ is the reciprocial time for scattering particles out of the trapped region; and $w_{b e}$ is the electron bounce frequency. 


\section{THIS PAGE}

\section{WAS INTENTIONALLY \\ LEFT BLANK}




\section{GOVERNING EQUATIONS AND APPROXIMATIONS}

Both the time evolution of a plasma discharge in a toroidal confining field and the quasi-equilibrium state to which the plasma evolves are governed - for sufficiently slow changes - by the driftkinetic, Fokker-Planck equation. The underlying physics forming the basis for this equation has been the subject of discussion for many years, and increasingly sophisticated derivations have been offered,

perhaps the most notable being that given recently by Hazeltine. ${ }^{13}$ This equation, governing the (guiding center) distribution functions of the various species in a plasma, has been written in a variety of different coordinate systems, both for velocity- and configuration-space. For analytic studies of plasma behavior in tori, the flux-energy magnetic moment description has proved effective and popular. However, for use in the CPM, in which sets of orthogonal transcendental functions are used in the description of velocity space, it becomes convenient to use scalar velocity and pitch-angle variábles in the velocity-space description. The rationale for and characteristic features of this approach are described in detail in Ref. 11. The transcription from one coordinate system to the other is tedious but straightforward. (Of course, the use of pitch-angle, scalar-velocity variables does not preclude employing flux coordinates in ordinary space.)

The drift-kinetic, Fokker-Planck equation in the CPM formulation is written out conveniently in considerable generality in Refs. 11 and 12. In the one paper, ${ }^{11}$ attention is focused in the Fokker.-Planck collision operator in the CPM description, showing that it can, in this case, be treated exactly, while in the second paper ${ }^{12}$ the spotlight is. 
on the drift-kinetic version of the vlasov operator. In Ref. 12 this part of the equation is treated in sufficient generality to allow for time-varying (self-consistent) fields and for a quite accurate description of the particle drifts.

In the present paper, however, we limit ourselves to a report of results obtained under the restrictions listed in Section I. Since the ions are treated as a stationary background, we require a kinetic equation only for the electrons. The confining magnetic field and the induction electric field are given by $\vec{B}=B_{0}(\hat{\zeta}+\hat{\theta} \varepsilon / q) /(1+\varepsilon \cos \theta)$ and $\vec{E}_{\zeta}=\hat{\zeta} V /\left[2 \pi R_{0}(1+\varepsilon \cos \theta)\right]$, where $\varepsilon=r / R_{0}$. The assumption of a specified and time-invariant $\vec{B}$ is tantamount to using the PfirschSchlüter divergence-free model field and actually implies the same low- $B$ approximation. We also neglect $B_{\theta}$ in the $\Omega^{-1}$ drift terms, where $\Omega$ is the electron gyrofrequency. In this way, the general equation in Ref. 12 (Eq. 3.6, with $\vec{V}=\vec{H}=G=0$, and $\hat{n} \equiv \vec{B} /|\vec{B}|$ ) simplifies substantially and is reduced lo

$$
\begin{aligned}
\frac{\partial \bar{f}}{\partial t} & +\left[v \cos \alpha \hat{n}-\frac{v^{2}\left(1+\cos ^{2} \alpha\right)}{2 \Omega_{\zeta} B_{\zeta}} \hat{\zeta} \times \vec{\nabla} B_{\zeta}\right] \cdot \vec{\nabla} \bar{f} \\
& +\hat{n} \cdot\left[\frac{v^{2} \sin \alpha}{2 B_{\zeta}} \vec{\nabla} \hat{B}-\frac{e}{\bar{M}_{e}} \vec{E}_{\zeta} \hat{n}\right]: \vec{\nabla}_{v} \bar{f}=\bar{C} \bar{f} .
\end{aligned}
$$

The clectron guiding center distribution $\bar{\Gamma}(\vec{x}, v, u, t)$ is expanded as suggested in the CPM description (Eq. (4.3), Ref. 12) so that Eq. (II.1) becomes a matrix equation for the time evolution of the various amplitudes making up $\bar{f}$. 
In the present study a radial localization approximation is further imposed on Eq. (II.1). Specifically, we assume that

$$
\bar{f}(r, \theta, v, \alpha, t) \cong N_{0}(r)\left[\frac{\rho(r)}{2 \pi}\right]^{3 / 2} \mathfrak{f}(\theta, \sqrt{\rho} v, \alpha, t),
$$

where $\rho=M_{e} / k T_{0}$, and $N_{0}$ and $T_{0}$ are the $t=0$ electron density and temperature, respectively. We further specify the radial gradients $r / N_{0}\left(d N_{0} / d r\right)$ and $r / T_{0}\left(d T_{0} / d r\right)$ entering in Eq. (II.I) as parameters related directly to moments of $\bar{f}$. More precisely, to the extent that $\exists$ by assumption has no explicit r-dependence, Eq. (II.2) implies $\bar{f}$ to be (approximately) a local Maxwellian, provided we take $\mathrm{N}$ and $\mathrm{T}$ to be the local density and kinetic temperatures at $t=0$. Then, neglecting $\partial \tilde{\mathrm{f}} / \partial \mathrm{r}, \partial \overline{\mathrm{f}} / \partial \mathrm{r}$ is entirely specified when $\mathrm{dN}_{\mathrm{o}} / \mathrm{dr}$ and $\mathrm{dT} / \mathrm{dr}$ are, and the kinetic equation can then be solved locally as long as $\bar{f}$ remains approximately a local Maxwellian.

The price paid for this localization is that $\bar{f}$, and hence various observable quantities, has an inherent error proportional to the nonMaxwellian part, or, alternatively, to the difference at each $(r, \theta, t)$ between $\mathrm{N}_{0}$ and the actual local density and between $\mathrm{T}_{0}$ and the actual Inral kinetir. temperature. Physically, the idoa of trcating the plasma locally arises from the notion that communication is much more rapid along field lines than across them, and for this reason individual flux surfaces (when they exist) tend to be isolated. It is true that transport (inter-particle transfer) of virtually all quantities of physical interest is much less restricted parallel to $\vec{B}$ than across it. But even in the time-independent problem, nonlocal phenomena arise, at the very least as the result of the radial component (more precisely, the component 
aligned with $\vec{\nabla} \psi$ ) of the momentum balance equation. A familiar example of this type of phenomenon, even in the completely static case, is the Shafranov shift, ${ }^{14}$ which relates the distortion of flux surfaces to the pressure gradient and plasma-loop inductivity in a finite- $\beta$ analysis. Thus, when the plasma currents and magnetic field are coupled self-consistently, the shape of the flux surfaces depends not only on the pressure gradient but also on the total current flowing within $\psi$, and in this sense is nonmlocal.

of course, in the present study, using a fixed model field, the above phenomenon is not included and we expect that the time-independent pressure balance within such an approximation can again be interpreted localiy. For this and other reasons, our time-asymptotic results ought to be satisfactory and comparable to earlier work, ${ }^{6,8,9}$ at least within the applicability of the use of the model $\vec{B}$-field. By contrast, the CPM time-dependent analysis, at its present stage of development, is very likely to be much more affected by the local approximation. This possibility is discussed in Section III.A in the light of our actual initial-value results.

As we shall see, the extent of the errors involved with the local approximation depends on the time scales involved and on the physical quantity of concern. In the high collisionality regime, in which $\bar{f}$ is constantly driven toward a local Maxwellian, we might at first expect the approximate replacement Eq. (II.2) to apply best. The same argument would lead one to suspect that conservation laws involving lower moments might be better satisfied than those involving higher moments, since low-energy particles (of higher collisionality) are more important in the former than in the latter. 
On the other hand, in the time-dependent problem, the plasma dynamics intrinsically involves non-local effects, not only through the radial momentum equation, but also through the angular momentum balance. Roughly, the angular momenta on different flux surfaces are coupled both through cross-field mass flux and also through centrifugal and coriolis forces. This can lead to the development, for example, of poloidal spin-up; ${ }^{15-19}$ this phenomenon is non-local ${ }^{19}$ and has its own characteristic time scale. ${ }^{16}$

Thus, competing with the argument that higher collisionality 1mproves the local approximation (as we use $i t$ ), by keeping the distribution closer, to a local Maxwellian, is the fact that cross field diffusion is more rapid at the higher $\nu_{*}$ 's than at lower ones, thus leading to the idea that a given flux shell will be more isolated, dynamically, for the banana regime than for the Pfirsch-Schlüter regime.

If the local approximation is in fact a good one, i.e., if both arguments above were to turn out to operate in our favor, then taken together, they could suffice to justify the use of localization over a very wide range of $\nu_{*}$, indicating at the same time its limitations and how and when it might break down. In this connection we remark that insofar as $\bar{f}$ itself is well calculated on a shell, the corresponding fluxes $\left(\Gamma_{r}, Q_{r}\right.$, etc.) will also be well calculated; however, quantities such as $\langle\dot{N}\rangle,\langle\dot{T}\rangle$, etc., will tend to be less accurate since they depend in part on a radial derivative $\partial \tilde{f} / \partial \mathrm{r}$ which has been neglected. 
The localization restriction is presently in the process of being relaxed in the CPM, and eventually it will clearly have to be removed entirely if we wish to be certain of a completely reliable description of the actual time evolution of a toroidal discharge toward its timeasymptotic state. 


\section{NUMERICAL RESULTS}

In this section we discuss results obtained by the means described in Section II for both transient phenomena and for various physical quantities in their eventual quasi-equilibrium states. These numerical results have been obtained over a wide range of collisionalities, from $v_{\star}=0.1$ (banana regime) to $\nu_{\star}=100$ (Pfirsch-Schlüter regime). The value of $v_{*}=.1$ indicates, of course, that a typical trapped particle can successfully traverse its bounce (or banana) orbit roughly

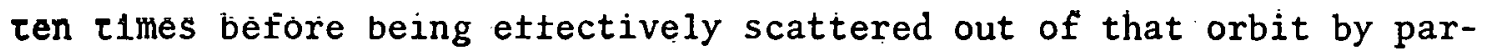
ticle-particle interaction. Conversely, a value of $\nu_{*}=100$ indicates that a particle that would be trapped in the absence of collisions has very little chance of traversing its assigned bounce orbit before being scattered away by its neighbors; correspondingly, we expect the distribution to show very little structure identifiable with particle trapping in this case.

Although it is very well known, it bears repeating here that even at high collisionality (e.g., $\left.\nu_{\star}=100\right)$ the actual mean-free-path based on $90^{\circ}$ Coulomb scattering for a freely circulating thermal particle at or near fusion temperatures is so long that such a particle would actually travel completely around a typical tokamak several times before being scattered. This fact calls into doubt the very concept of transport coefficients (resistivity, viscosity, thermal conductivity, etc.). We are somewhat redeemed in their use for the present application, however, by the fact that trapped particles are extremely important, and that these particles experience an exaggeratcd 
effect of collisions. We are further helped by the fact that many present methods of starting up toroidal discharges present us, at least initially, with distributions which are approximately Maxwellian. Finally, with regard to the very important cross-field transport, it is not really the classical Spitzer mean-free-path, but rather the mean gyro-radius, which is representative of the inter-particle communication length, and this in turn establishes cross-field transport of macroscopically observable quantities. For this reason, it turns out to rcmain useful to think in terms of transport phenomena, at least in the cross-field sense; even at low collisionality. 20

\section{A. Transient Phenomena}

Our original intention in studying the initial value problem described earlier was simply to use it as a numerical method of allowing a toroidally-confined plasma to achieve a quasi-steady state. In this scheme the time-asymptotic states calculated by the code are then to be taken to represent the tokamak equilibria which are to be perturbed later for stability studies, particularly in the nonlinear analysis of trapped-particle modes. However, this time-dependent work itself provides a considerable amount of useful information concerning the gross dynamical behavior of a toroidal plasma discharge as it develops In tlme. This infurmaliun ban be didalyed in the light af variona theoretical treatments, which have become available over the past several years, ${ }^{15-18}$ of the initial value problem for a toroidal discharge, particularly at moderate to high collisionalities. Conversely, the transient results obtained in this way are expected to be very 
useful: in guiding the much more difficult treatment of the corresponding initial value problem at low collisionality 21.

Present results for the transient problem are summarized in

Figs. 1, 2 and 3. Figure 1 shows plasma behavior at high and low values of $v_{*} \cdot T_{0}$ has been kept constant, but $N_{0}$ differs for different $v_{*}$, as indicated. In Figs. 1a-1d, time development of high-temperature discharges is illustrated in these various cases for situations with radial density gradients only, and with radial density and temperature gradients working together. In Figs. $2 \mathrm{a}$ and $2 \mathrm{~b}$ a lower temperature case is shown for $\nu_{*}=100$ with $\mathrm{r} / \mathrm{N}_{\mathrm{o}}\left(\mathrm{dN}_{\mathrm{o}} / \mathrm{dr}\right)=-2$. Time development of this discharge is compared for two situations; without and with an applied ring voltage. Figure 3 shows a comparison of times to attain equilibrium with different effective $Z$ for the ions.

The reader will note that the time plots are given in units of collision time at differing $\nu_{*}$, so that corresponding points on the abscissae of the various graphs represent different real times. A useful rule of thumb, however, is provided by noting that if a nominal experimenta1-duration time is taken to be $50 \mathrm{msec}$, then the time to attain quasi-equilibrium (defined in Section III.B) is always somewhat longer than, but nearly equal to, only one such experimental-duration time. The quantities monitored on the graphs are the radial mass flux, $\Gamma_{r}$; the radial heat flux, $Q_{r}$; the parallel current density $J_{\|}$; the fractional temperature change $\delta \mathrm{T}_{\mathrm{e}} \equiv\left(\mathrm{T}_{\mathrm{e}}-\mathrm{T}_{\mathrm{o}}\right) / \mathrm{T}_{0}$; and the poloidal and toroidal mass-flow velocities $V_{\theta}$ and $V_{\zeta^{\circ}}$ 'All quantities are fluxsurface averaged with the exception of $v_{\theta}$ and $v_{\zeta}$, which are calculated 
at the outside of the torus $(\theta=0)$. The units in terms of which the quantities are measured are $N_{0} \sqrt{3 / \rho}$ for $\Gamma_{\mathbf{r}}$ and $J_{\|}, 1 / \sqrt{\rho}$ for $v_{\theta}$ and $v_{\zeta}$, and $(3 / 2) \mathrm{M}_{\mathrm{e}} \mathrm{N}_{\mathrm{o}} \sqrt{\rho^{3}}$ for $\mathrm{Q}_{\mathrm{r}}$.

It will strike the reader immediately that, for several of the cases shown, a variety of time scales is present. The early, fastest time scale occurring for each $\nu_{*}$ is evidently the time required for the initial Maxwellian distribution to make its preliminary adjustment to the particle trapping occurring in competition with collisions. The fact that this initial time is about the same proportion of the overalt transient required in establishing the quasi-equilibrium (compare Figs. 1a-b with Figs. 1c-d) is consistent with the definition of $v_{*}$ and/or with the relative importance of the trapped particles in the various regimes. This phenomenon is readily interpreted locally, i.e., as one occurring on an essentially isolated flux she11. It has nothing to do with the overall dynamics of the discharge as discussed, for example in Ref. 15, for high $\nu_{\star}$. Indeed, it is much too fast, and requires a kinetic theory trcatment. 21

In Fig. 1a, however, additional time structure is evident in the later phases of relaxation toward quasi-equiliprịm. The question arises at once as to whether these time scales can be explained at high $\nu_{\star}$ in terms of available dynamical theories such as those cited (Refs. 15-18). The absence of any comparable time structure in Fig. 1b, in which an initial temperature gradient has been specified but otherwise all conditions remain the same, suggests an alternative explanation, to be discussed below. 
One way of checking whether the time scales evident in Fig. 1a are true dynamical ones is to compare Figs. $1 \mathrm{a}$ and $2 \mathrm{a}$. These two cases are comparable in every respect, except that $\mathrm{T}_{0}$ has been changed by a factor of $\sim 30$ with $\nu_{*}$ held constant: Since $\nu_{*}$ scales as $\mathrm{N}_{0} / \mathrm{T}_{0}^{2}, \mathrm{~N}_{0}$ changes between the two figures by a factor of $\sim 10^{3}$. Now, the dynamical times in the theories of Refs. $15-18$ scale as $\mathrm{T}_{0}^{1 / 2} / \mathrm{N}_{0}$, whence these time scales, in units of the electron-ion collision time $\tau_{\text {ei }}\left({ }^{n} \mathrm{~T}_{0}^{3 / 2} / \mathrm{N}_{0}\right)$, should appear with a $\mathrm{T}_{0}^{-1}$ scaling in comparing Figs. 1 and 2.2 Clearly, this is not the case. Instead; the time scales remain the same in units of $\tau_{e i}$. This strongly suggests that the time structures we see in our transient calculations àre all (locảl) flux-shell readjustment times, just as was the fast transient. We believe the time structure in Fig. la is governed by the time required for the heat-flux vector, $Q$, to come to a quasi-equilibrium with the plasma. [It clearly has difficulty in doing so when no initial temperature gradient is present (Fig. la vs Fig. 1b).]

Note in Fig. $1 b$ that the mass flux $\Gamma_{\mathrm{r}}$ levels off to a nearly constant value very quickly after the initial fast transient. In view of the fact that these calculations are made with a given magnetic field and essentially with a constant temperature or energy density, this result is consistent with the known results for mass flux diffusion corresponding to the Pfirsch-Schlüter phenomena associated with the basic toroidal geometry. In that sense; therefore, the quick flattening of $\Gamma_{r}$ is a verification of the accuracy of the computer program. Also, a comparison of $\dot{\Gamma}_{\mathrm{r}}$ for the 'low and high temperature Pfirsch-Schlüter results verifies that it scales properly, which in the units used is simply as $\mathrm{T}_{0}$. 
For our calculations, a quantity of great importance is the heat flux vector. The reader will note that its behavior is quite different both in time and in terms of its asymptotic value depending on whether or not an initial temperature gradient is present; significantly, this fact is true for both large and small values of $\nu_{\star}$. This demonstrates that the heat flux is sensitive to the temperature gradient and is in this sense determined at least time-asymptotically by a transport phenomenon even at low collisionality. It is further evident that the heat flux vector has great difficulty in coming to equilibrium with the plasma when no initial temperature gradient is provided. Consistent with our interpretation, given above, of the observed time variations being local, in the sense already described, we believe that the time structure evident in Fig. la is to a large extent simply imposed on the other quantities through the time variation of $U_{r}$ as it seeks an equilibrium with the discharge. This seems to be verified in Fig. $1 b$.

Hanally, we note that Figs. 1s and d are disu culisislenl will our interpretation of the observed time variations being strictly local readjustment times on an isolated flux shell. At low collisionalities such times scale with the bounce time, or, in units of $\tau_{e i}$, directly proportionally to $v_{*}$. Since this time is faster than the collision time, the time to attain equilibrium is independent of $\mathrm{d} \mathrm{i} / \mathrm{dr}$ and depends on the time needed for collisions to ameliorate the fast transients.

The effect of the modest ring voltages which have been applied in some cases is consistent to the extent that it leads to an increase in the total current flow (especially the current flowing parallel 
to the magnetic field) and the temperature: Figure $2 b$ shows this heating occurring when a ring voltage is applied. Because of the almost linear dependence of $\mathrm{T}_{\mathrm{e}}$ on time, this plasma appears to be in a runaway state. The phenomenon of joule heating cannot be adequately studied locally, since it is influenced by radial temperature gradients and heat fluxes, which also depend strongly on ion dynamics. In addition, the fact that collisionality is a strong function of temperature strongly affects the study of the heating problem. 22 Furthermore, the voltage which would, in the real experiment, drive the current necessary to establish the poloidal field is not, in these calculations, consistently connected with that poloidal field; i.e., Maxwell's equations have not been self-consistently applied. In order to do this we would require a self-consistent calculation of the time-varying magnetic field as related to the actual current flowing through the plasma. This self-consistent initial value problem has been shown ${ }^{19}$ to be important for high collisionality.

In order to make our calculations self-consistent, we see that we not only need to include the time variation of the magnetic field, which is related, of course, to the current induced in the plasma by the ring voltage, but we also need to relax the local approximation. To make the problem numerically tractable, we must allow for variation in the cemperature consistent with development of a heat flux and with the joule heating occurring when a ring voltage is applied. In our plans for including this temperature consistency we must also include a variation of $\nu_{*}$ during the time evolution of the plasma discharge. 
Nevertheless, the present results provide useful insight into the temporal behavior of toroidal plasma discharges and in fact suggest methods by which a self-consistent calculation can be carried out. Perhaps more significant for our present circumstances, however, is the fact that we expect the quasi-equilibrium, discussed in the next section, to be valid, even though the initial transient by which it is obtained is almost certainly not completely self-consistent. This is because we have chosen our initial values of density, temperature, $\nu_{\star}$, the relcvant gradients, etc., as well as the confining magnetic field, to be consistent with their expected equilibrium values in the timeasymptotic state.

\section{B. Neoclassical Equilibria}

As stated before, the equilibrium calculated here is really a quasi-equilibrium in which the only change is a slow radial diffusion. Since, for a given radial density gradient, this diffusion is proportional to the number density, the diffusion will result in an exponential dependence of $\mathrm{N}_{e}$ on time. Therefore, $\partial^{2}\left(\ln \mathrm{N}_{\mathrm{e}}\right) / \partial \mathrm{t}^{2}$ will approach zero. This also implies that $\Gamma_{r}$, the radial flux, becomes almost constant in time. We use either of these two criteria to determine whether we have indeed reached a quasi-equilibrium.

Past work has tended to divide the regimes of collisionality into high (Pfirsch-Schlüter), medium (plateau) and low (banana): As far as characteristic behavior goes, there are really only two regimes: high and low collisionality. The remaining regime is simply a transition between the two limiting regimes. (It might be remarked that this transition regime - the most difficult to handle analytically - is easiest to 
treat numerically.) In discussing our results, we concentrate principally on the limiting cases.

We have systematically studied equilibria (over all collisionalities) in a number of different cases, four of which are discussed here; these cases are characterized by specifying that

1) a radial density gradient exists alone,

2) radial density and temperature gradients exist together,

3) a radial density gradient and an induction field $\left(E_{\dot{\zeta}}\right)$ exist together,

4) a radial density gradient exists alone with high-Z ions.

In showing distribution functions $\bar{f}$ which are almost entirely the initial Maxwellian, the Maxwellian component $\overline{\mathrm{f}}_{\mathrm{m}}$ should be subtracted from the entire distribution. All figures will therefore show the difference $\hat{\mathrm{f}}=\overline{\mathrm{f}}-\overline{\mathbf{f}}_{\mathrm{m}}$. These functions $\hat{\mathbf{f}}$. are shown in three-dimensional plots in Figs. 4 and 5 for high and low collisionality both without and with a radial temperature gradient. The $v_{\|}$axis is in the direction of the magnetic field $\vec{B}$. The $v_{\perp}$ axis origin is on the side with the largest function value, in the center of the $v_{\|}$axis. It should be remembered that this $\hat{\mathbf{f}}$ represents a slice through a cylinder in velocity space, and that to understand the figures in terms of the particle density at a given $\left(v_{\|}, v_{\perp}\right)$, one must multiply $\hat{f}$ by $v_{\perp}=v \sin \alpha$.

In the case in which there is only a radial density gradient, the dominant characteristic of $\hat{f}$ is that it produces a current which varies as $\cos \theta$. However, we find that this current is not produced by a shifted Maxwellian; this can be verified by a detailed examination of the Fourier components of the $I_{\text {ln }}$ amplitudes (see Eq. (4.3) in Ref. 12) and 
by. the $\theta$-dependence of macroscopic observables. Moreover, the picture of $\hat{\mathrm{f}}$ at $\theta=90^{\circ}$ (Fig. 4a) clearly shows a non-Maxwellian perturbation corresponding to a local decrement in temperature. We conclude that the flow (which at $\theta=0^{\circ}$ and $\theta=180^{\circ}$ is anti-parallel and parallel to $\vec{B}$, respectively) is characterized more by gradients than by a drift of purely collisional particles.

Just as particles at sufficiently high energy behave collisionlessly in the collisional regime, so low energy particles in the collisionless regime must behave collisionally. The difference is that in the collisional regime, these anomalous particles are a vanishingly smali part of the Maxwellian tail, while in the banana regime, they occupy the part of velocity space which is most heavily populated. Therefore, for $\theta$ such that the collisional and collisionless particle currents are in opposite directions $\left(\theta=180^{\circ}\right.$, inside of torus) the distribution function must exhibit a great deal of structure, as is shown in Fig. 4 b. We may compare this result with results from earlier and more approximate calculations. The calculations of Tsang and Callen ${ }^{9}$ apply to a high-Z equilibrium in the Pfirsch-Schlüter regime for $\nu_{*}=100$ and are shown in Fig. 6a, while those of Rosenbluth, Hazeltine, and Hinton ${ }^{6}$ are applicable to the banana regime for $\nu_{*}=0$ and are shown in Fig. $6 \mathrm{~b}$. When a radial temperature gradient is present as well as a radial density gradient, the equilibrium distribution has considerably different characteristics (see Figs. $5 a$ and $5 b$ and compare with Figs. $4 a$ and $4 b$ ). One of the main differences is the difference in increase of temperature on the shell. With no radial temperature gradient there is a small overall increment of temperature on the she11, although there is actually 
a local decrement. at the top and bottom of the torus. With a temperature gradient, the flux-surface average temperature is larger by a factor of 30, and the temperature everywhere increases. This is true for all collisionalities. The phenomenon shows up in the distribution function as a decrease of $\hat{f}$ near zero velocity and an increase of $\hat{f}$ at moderate velocity: It is observed most clearly (Fig. 5a) in the Pfirsch-Schlüter regime, where the simple $\cos ^{\theta} \theta$ dependence of $J_{\|}$no longer obtains. It is further observed for $\nu_{\star}=0.1$ (Fig. 5b) with the vanishing of the low-energy spike at $\theta=180^{\circ}$. The further observation that this equilibrium is attained much more quickly (see Fig. 1b) than when $\mathrm{dT}_{0} / \mathrm{dr}=0$ indicates that a plasma with $\mathrm{dT}_{0} / \mathrm{dr}<0$ is closer to an equilibrium than a plasma.with an iverted or neutral radial temperature profile. This is further supported by the inward direction of the heat flux vector when $\mathrm{dT}_{\mathrm{o}} / \mathrm{dr}=0$, indicating that the plasma is attempting to establish a.state where $\mathrm{dT}_{\mathrm{o}} / \mathrm{dr}<0$.

In the calculations described above, we have kept $T_{0}$ constant as $\nu_{*}$ becomes large. For $\nu_{\star} \sim 1$, this corresponds to a tokamak-like density, but for $v_{*} \sim 100$, the density becomes unrealistically large. A more physical density obtains in this latter case if we takc $T_{0}=30 \mathrm{eV}$. We find that while: the magnitude of $\hat{f}$ varies with density and temperature, the shape of $\hat{f}$ is independent of these quantities, but depends only on $\nu_{\star}$. Thus, the case for $T_{0}=30 \mathrm{eV}$ and $\nu_{\star}=100$ yields three-dimensional plots identical to F1g. $4 \mathrm{a}$.

If we now include a ring voltage, we see the expected effects on both parallel current and temperature. In an actual experiment, the plasma current, and hence $B_{\theta}$, would be self-consistent with such a ring 
voltage. For our purposes, we simply.look at the effect during the time evolution, and in equilibrium, of a constant $E_{\zeta} \cdot$ We do this for a moderate-temperature $\left(\mathrm{T}_{0}=30 \mathrm{eV}\right)$ highly collisional $\left(\nu_{*}=100\right)$ plasma. (Studies at other $v_{*}$ give essentially the same result.) There is, of course, not much heating unless the electric field energy is comparable to the plasma energy. As the plot of $\delta T_{e}$ vs $V$ shows (Fig. 7), the joule heating certainly does occur. A similar plot of $J_{\|}$vs $V$ shows the strong effect of $V$ on the parallel current. This current and the heating are apparent in the distribution functions shown in Fig. 8. In a real tokamak, the ions would have an effective charge which is greater than unity. We have studied such a case, again in the high collisionality regime. The physical effect is that the electron-ion collision frequency is enhanced, and equilibrium is attained much faster (see Fig. 3). Otherwise, as before, the characteristics of the equilibrium distribution are the same as those of other $\nu_{*}=100$ cases.

We may compare our calculations with the results of previous calculations by Hazeltine and Hinton ${ }^{8}$ and Tsang and Callen ${ }^{9}$ by plotting $\Gamma_{\mathrm{r}}\left(\nu_{*}\right)$ for the three sets of calculations (Fig. 9), where the units of $\Gamma_{r}$ are those of Ref. 8. The earlier and more approximate results are shown to be in good agreement with the present results. 


\section{CONCLUSIONS}

The most important conclusion, which must be underscored, is that the present numerical procedure has been shown to be a viable method for calculating plasma equilibrium. In addition, the treatment of the initial value problem has yielded some new insights into the buildup of a tokamak equilibrium, even though some important physical effects may have been omitted.

Calculations are now underway to study the areas that must be listed as weaknesses in the present results. These areas are listed below.

1) Inclusion of ion dynamics in equilibrium calculations, especially to study the heat flow problem.

2) A study which includes the radial dimension and a timevarying temperature, to

a) verify or debunk the localization assumption, and

b) study equilibrium buildup correctly.

3) Inclusion of a self-consistent magnetic and toroidal electric field to provide a rigorous and correct study of a tokamak equilibrium.

4) Inclusion of self-consistent electric fields to study the dissipative trapped-electron instability. 
THIS PAGE

\section{WAS INTENTIONALLY LEFT BLANK}




\section{ACKNOWLEDGMENT}

We wish to thank $\mathrm{Dr}$. T. Uckan for making his numerical computations of the Rosenbluth-Hazeltine-Hinton and Tsang-Callen distribution functions available to us.

We are also greatly indebted to K. E. Rothe for a great deal of fine work in obtaining numerical results and in preparing them in a form suitable for presentation here. 
THIS PAGE

\section{WAS INTENTIONALLY}

LEFT BLANK 


\section{REFERENCES}

1. A. A. Galeev and R. Z. Sagdeev, Zh. Eksp. Teor. Fiz. 53, 348 (1967) [Sov. Phys. -JETP 26, 233 (1968)].

2. L. M. Kovrizhnikh, Zh. Eksp. Teor. Fiz. 56, 877 (1969) [Sov. Phys. -JETP 29, 475 (1969)].

3. P. H. Rutherford, Phys. Fluids 13, 482 (1970).

4. E. A. Frieman, Phys. Fluids $13,490 "$ (1970).

5. B. B. Kadomtsev and 0. P. Pogutse, Nucl. Fusion 11, 67 (1971).

6. M. N. Rosenbluth, R. D. Hazeltine, and F. L. Hinton, Phys. Flnida 15, $116(1972)$.

7. F. L. Hinton and M. N. Rosenbluth, Phys. Fluids 16, 8.36 (1973).

8. R. D. Hazeltine and F. L. Hinton, Phys. Fiuids 16, 1883 (1973).

9. K. T. Tsang and J. D. Callen, to be published in Phys. Fluids.

10. The approach referred to here is based on the particle simulation code described by K. T. Tsang, Y. Matsuda, and H. Okuda in Phys. Fluids 18, 1282 (1975).

11. H. K. Meier, W. I. van Rij, C. O. Beasley, Jr., and J. E. McCune, The Collisiona1 P1asma Mode1: A Velocity-Space Orthogona1 Function Representation for the Distribution Function of a Collisional Plasma, ORNL/TM-5314 (March 1976).

12. W. I. van Rij, H. K. Meier, C. O. Beasley, Jr., and J. E. McCune, Kinetic Equations for the Collisional Plasma Model, ORNL/TM-5316 (March 1976).

13. R. D. Hazeltine, Plasma Phys. 15, 77 (1973).

14. V. D. Shafranov, Rev. of Plas.Phys. Vol. 2, p. 103 (1969). 
15. R. D. Hazeltine, E. P. Lee, and M. N. Rosenbluth, Phys. Fluids 14, $361(1970)$.

16. R. D. Hazeltine, E. P. Lee, and M. N. Rosenbluth, Phys. Rev. Lett. $25,427 .(1070)$.

17. T. E. Stringer, Phys. Rev. Lett 22, 770 (1965).

18. P. W. Chrisman, Jr., "Inertial, Viscous, and Finite-Beta Effects in a Resistive, Time-Dependent Tokamak Discharge," Ph.D. Dissertation, Massachusetts Institute of Technology, Cambridge, MA (December 1975).

19. K. U. von Hagenow, J. E. McCune, and P. W. Chrisman, Jr., "ŚelfConsistent 'lime-Dependent MHD Toroidal Flow with Finite Resistivity and Viscosity," ERDA Doc. COO-3070-162; submitted to Nuc1. Fusion.

20. S. P. Hirshman, D. J. Sigmar, J. F. Clarke, accepted for publication in Phys. Fluids.

21. Jay Fischer (Massachusetts Institute of Technology), private communication, 1976.

22. K. T. Tsang and J. D. Callen, private communication, 1976. 


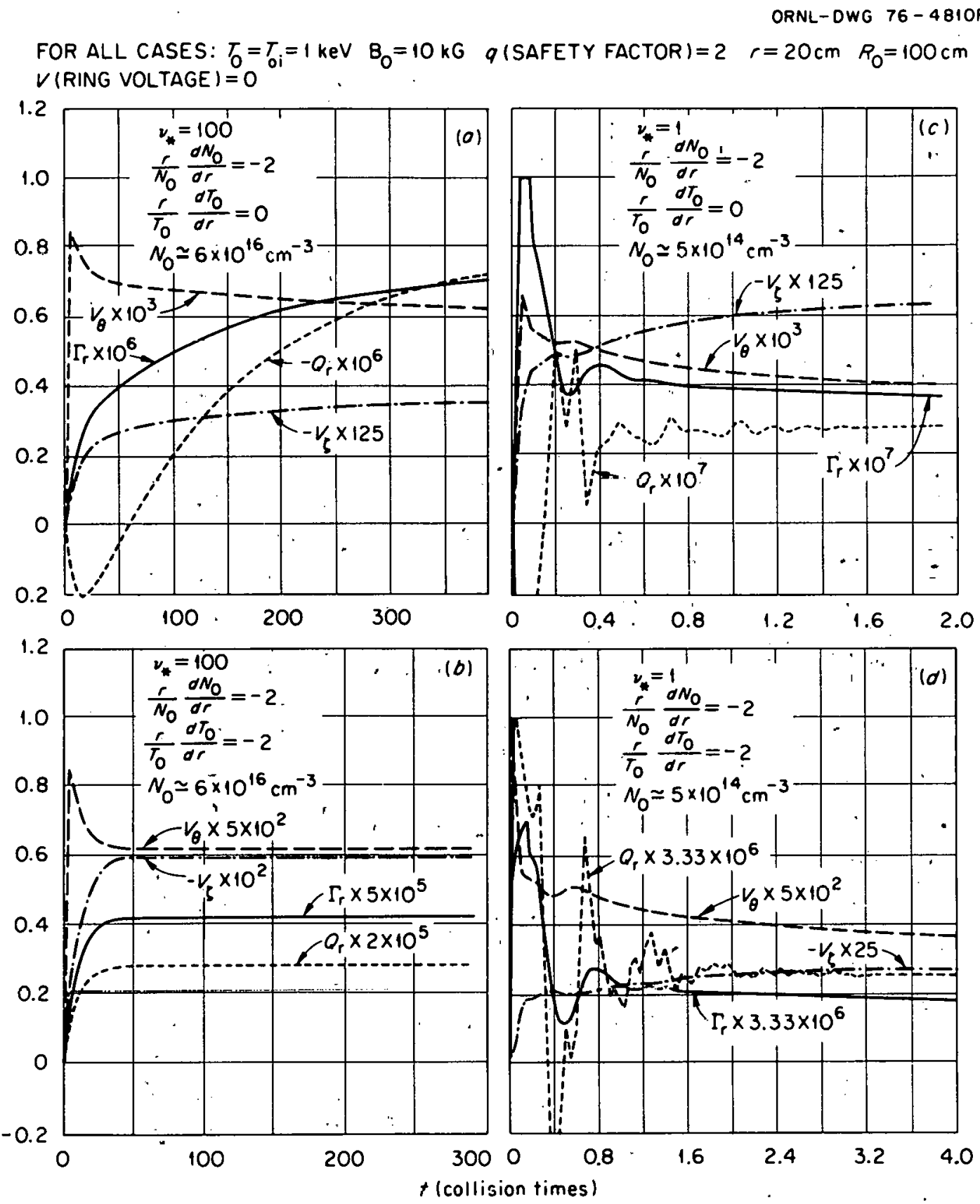

Time Evolution of a Tokomak Discharge.

Fig. 1 
ORNL-DWG 76-4809R

FOR BOTH CASES: $T_{0}=T_{0 i}=30 \mathrm{eV} \quad N_{0} \simeq 6 \times 10^{13} \mathrm{~cm}^{-3} \quad B_{0}=10 \mathrm{kG} \quad q=2$

$$
\nu_{*}=100 \frac{r}{N_{0}} \frac{d N_{0}}{d r}=-2 \frac{r}{T_{0}} \frac{d T_{0}}{d r}=0 \quad r=20 \mathrm{~cm} \quad R_{0}=100 \mathrm{~cm}
$$

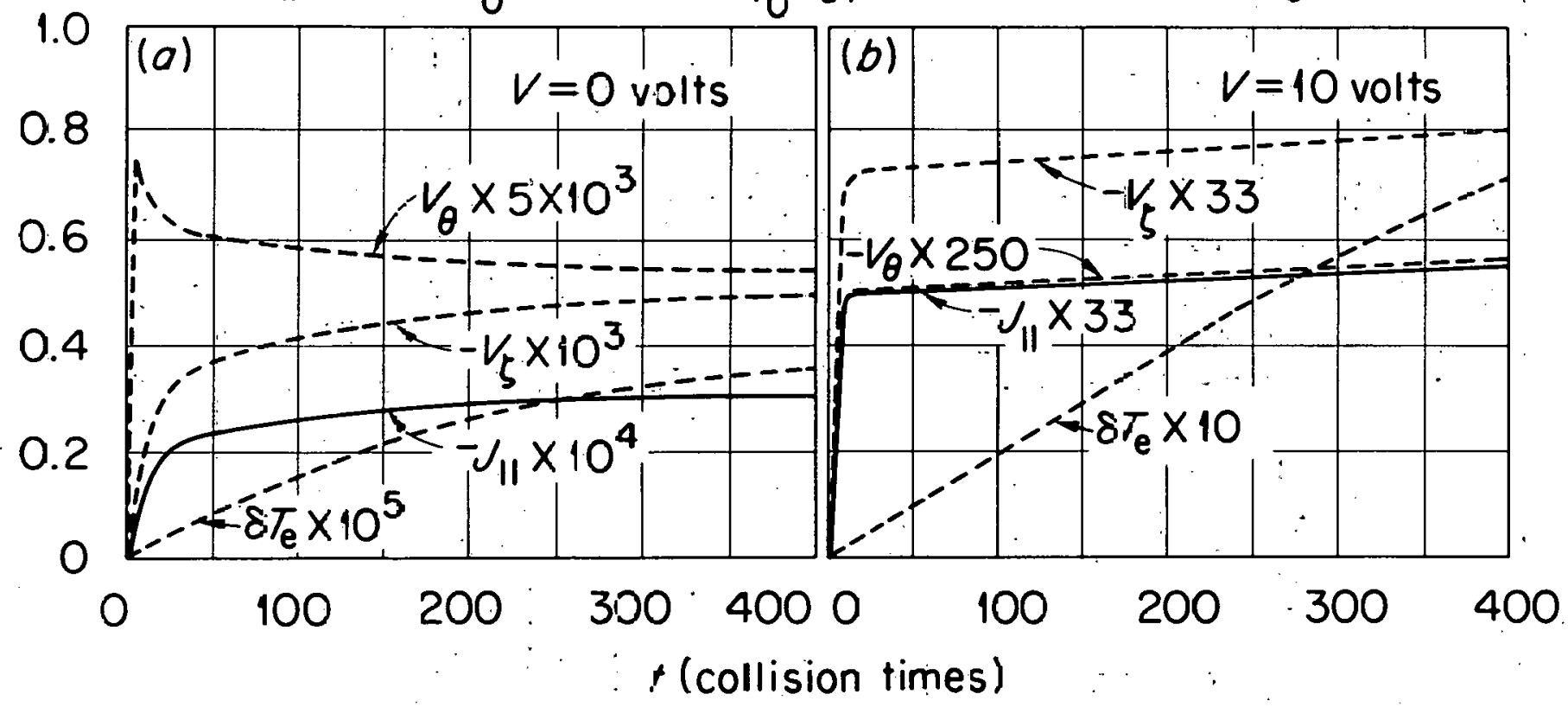

Time Evolution of a Low -Temperature, Highly Collisional Tokamak Discharge without and with a Ring Voltage.

Fig. 2 


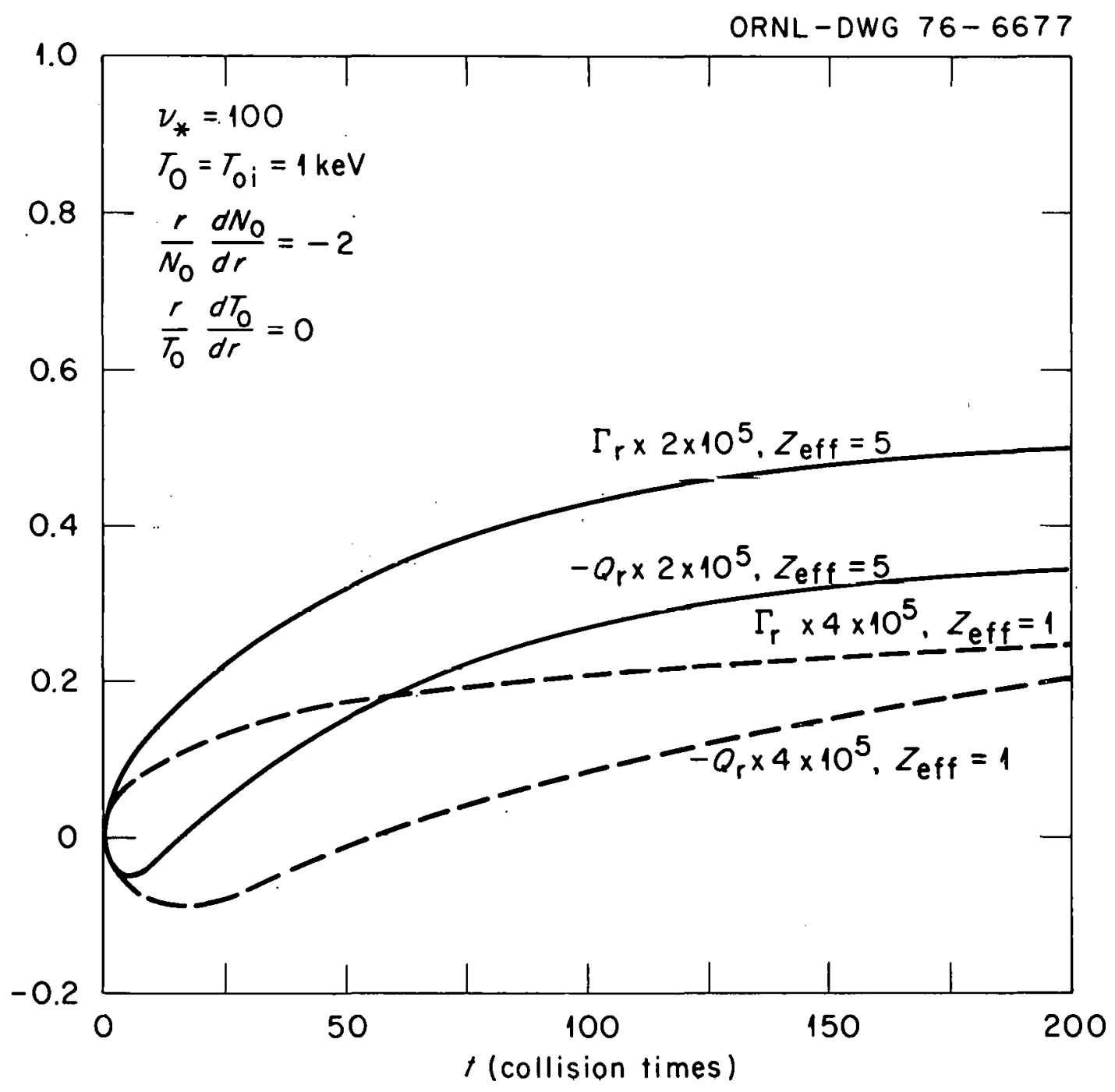

Comparison of Equilibrating Times for $Z_{\text {eff }}=1$ and $Z_{\text {eff }}=5$. Fig. 3 


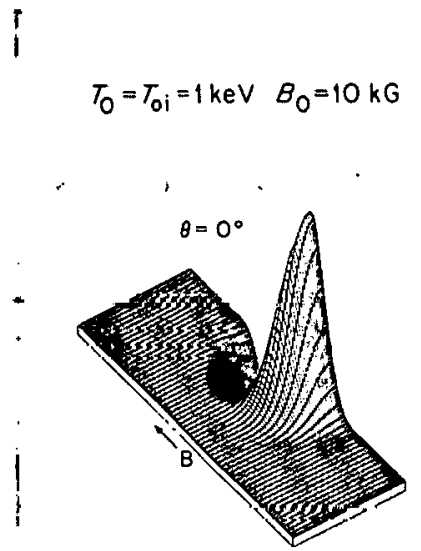

ORNL-DWG 76-4808A

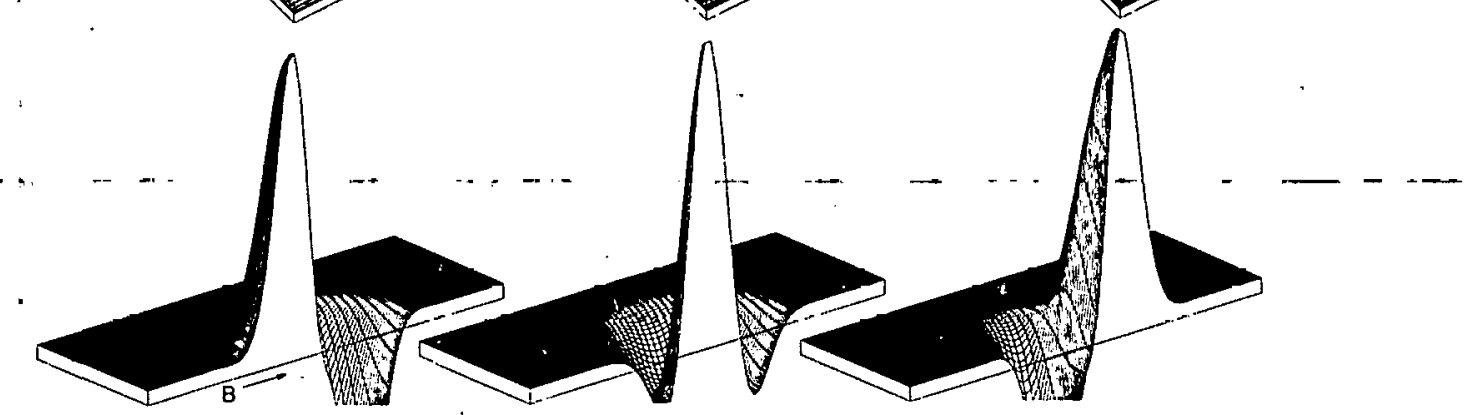

(a) $w_{*}=100\left(N_{0} \simeq 6 \times 10^{16} \mathrm{~cm}^{-3}\right)$

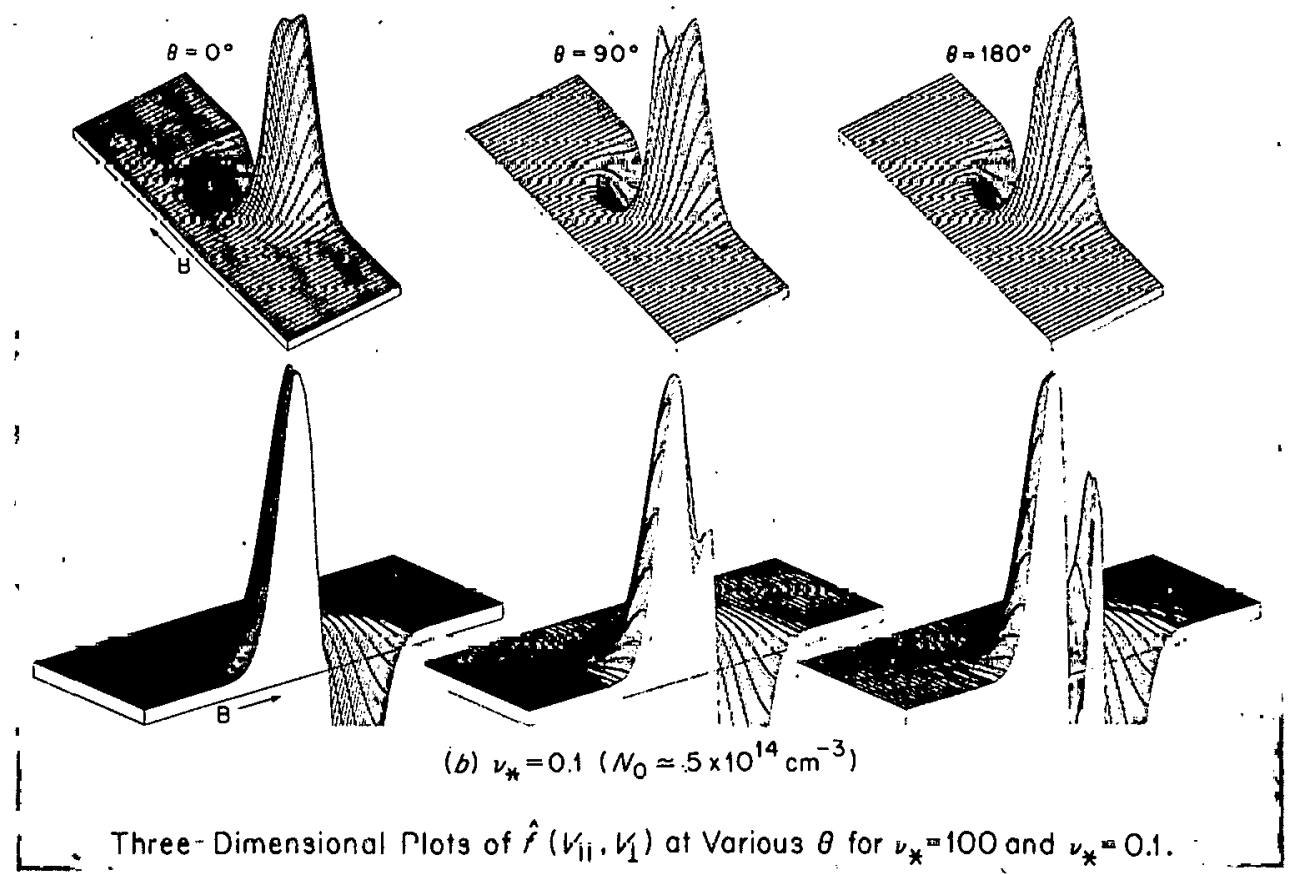

Fig. 4 

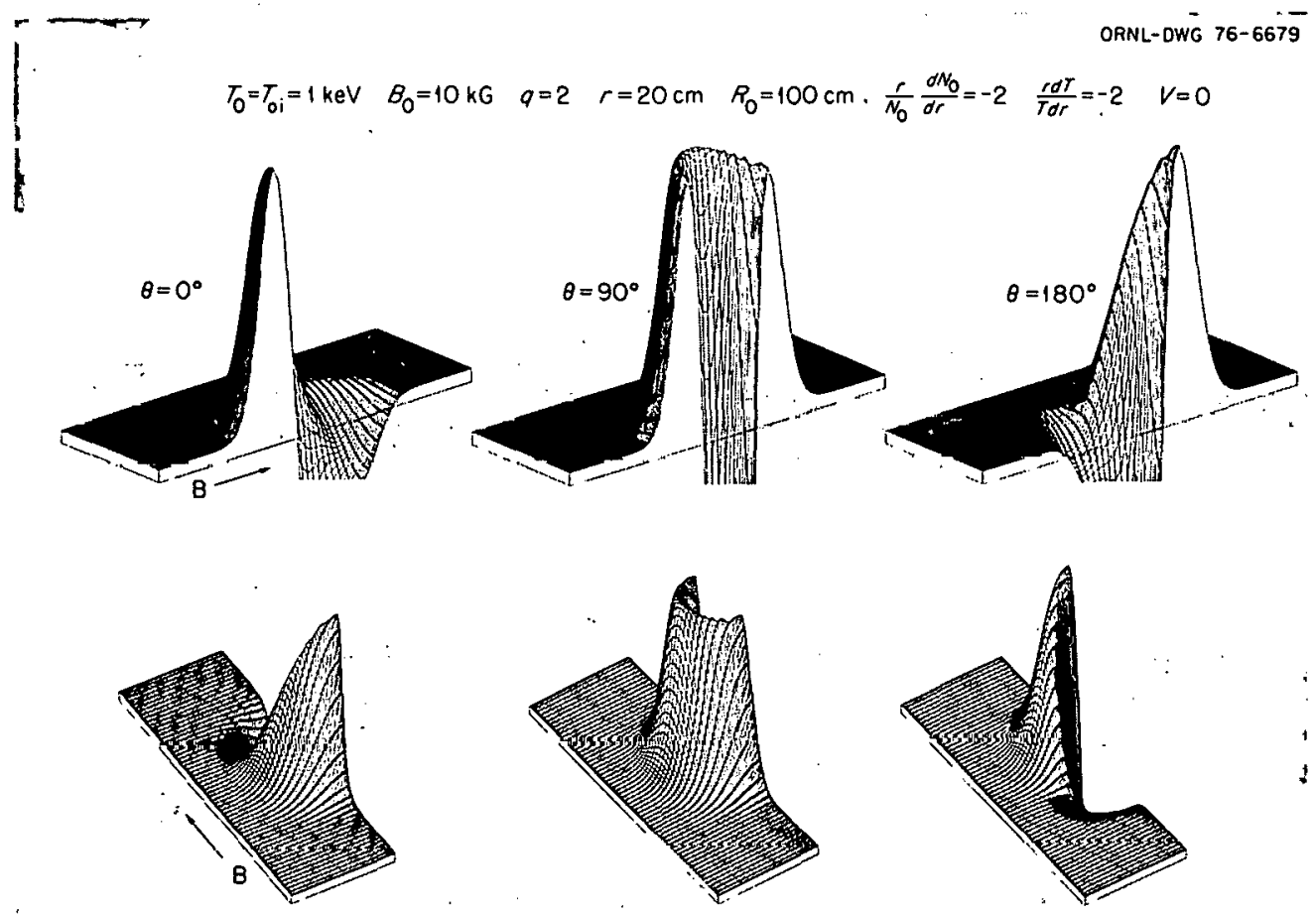

(a) $\nu_{0}=100\left(N_{0} \simeq 6 \times 10^{16} \mathrm{~cm}^{-3}\right)$
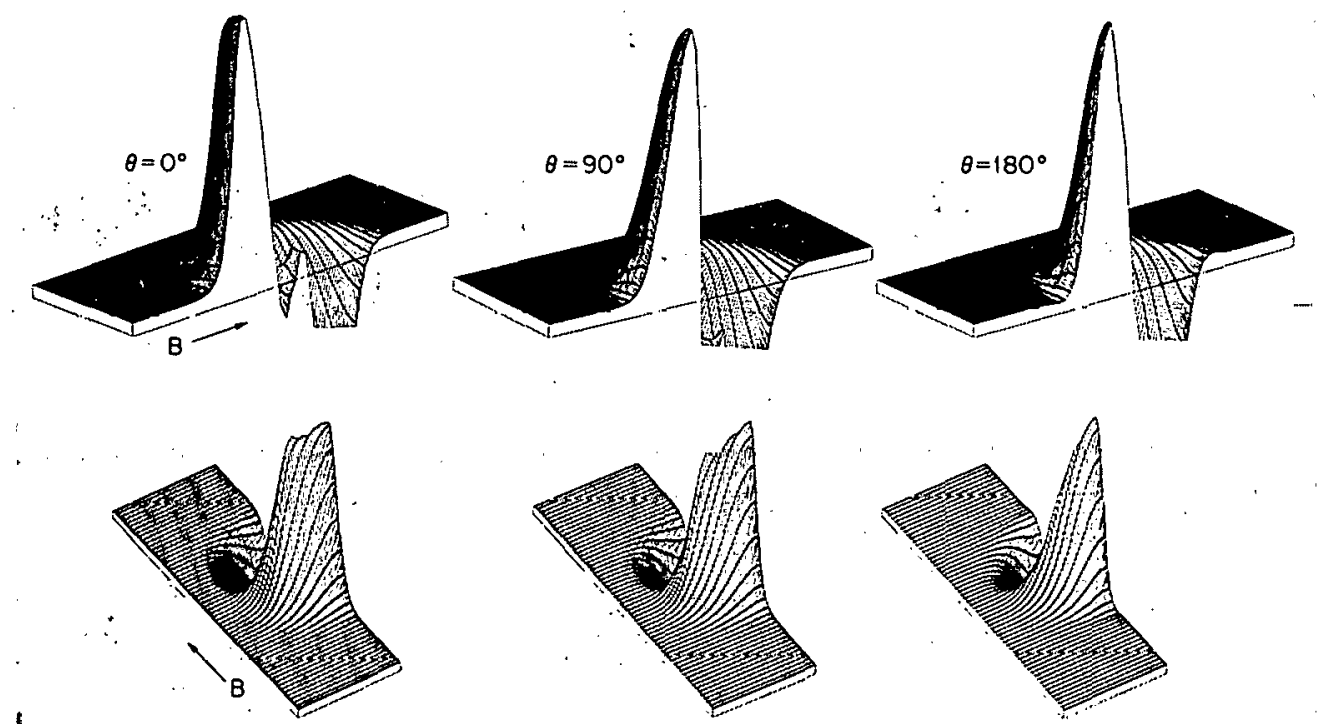

(b) $y_{*}=0.1\left(N_{0} \simeq 5 \times 10^{14} \mathrm{~cm}^{-3}\right)$

i Three-Dimensional Plots of $\hat{f}\left(V_{11}, V_{1}\right)$ at Various $\theta$ for $\nu_{*}=100$ and $\nu_{*}=0.1$.

Fig. 5 


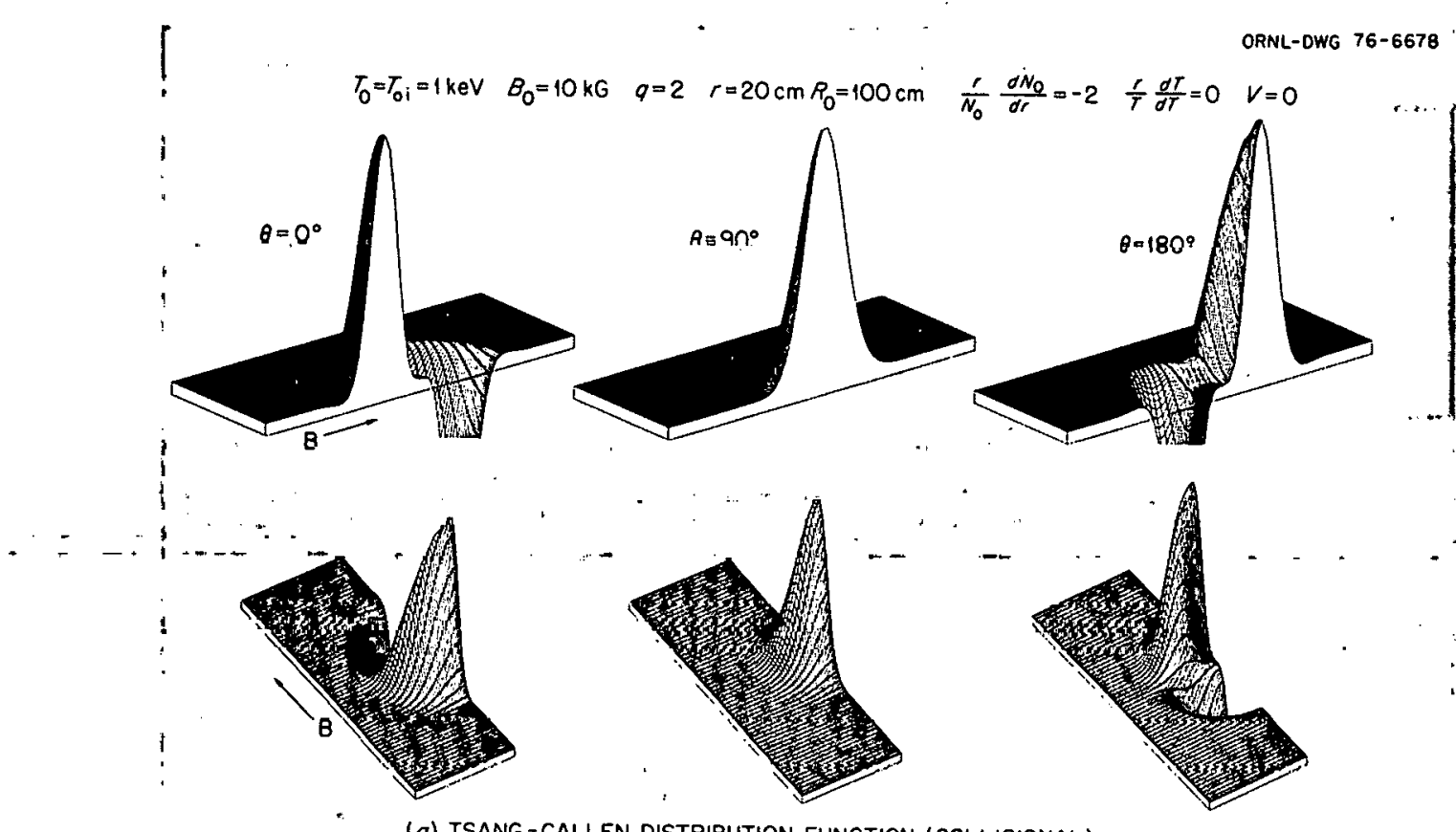

(a) TSANG - CALLEN DISTRIBUTION FUNCTION (COLLISIONAL)

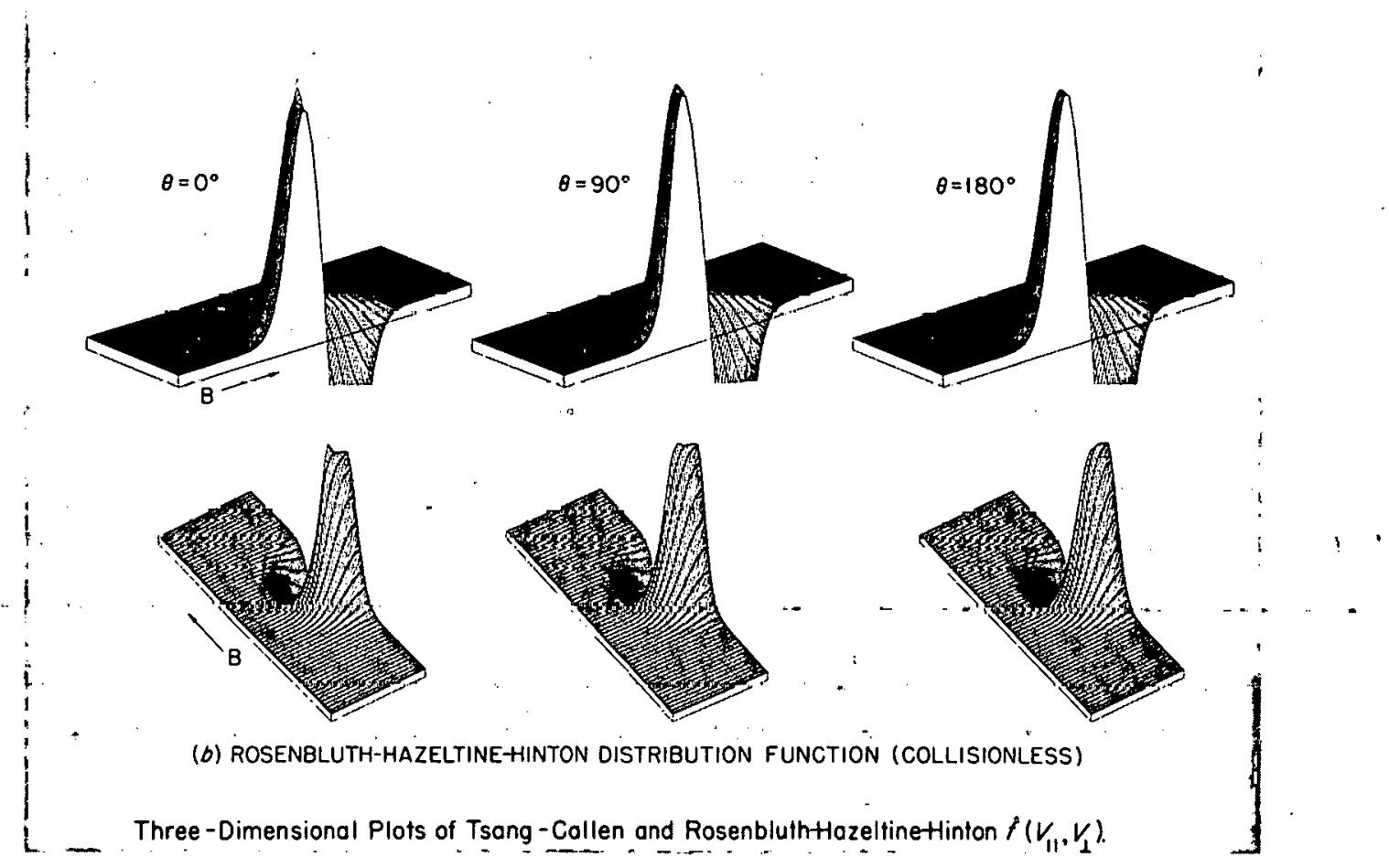

Fig. 6 


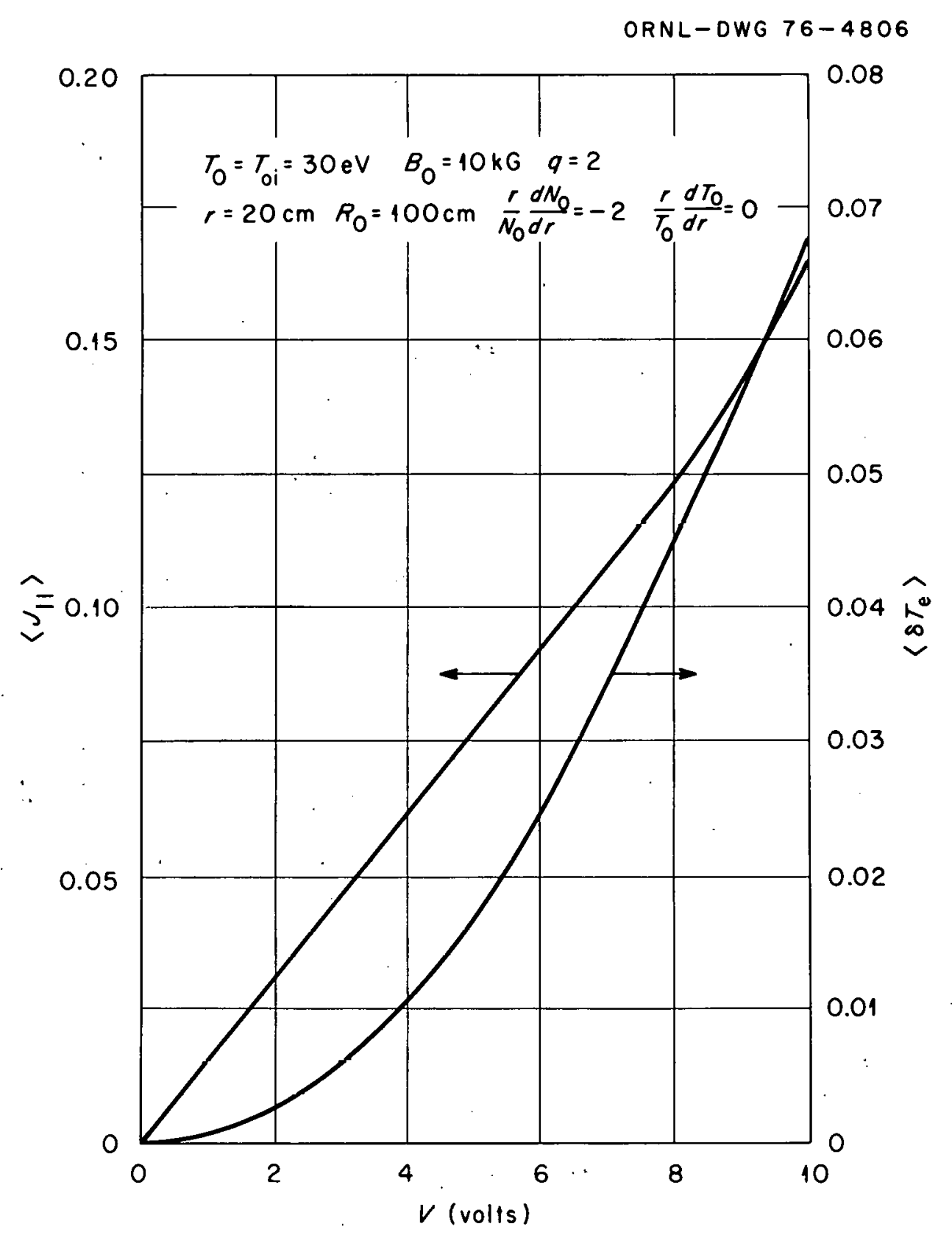

Flux - Averaged Parallel Current and Temperature Increase as a Function of Ring Voltage.

Fig. 7 


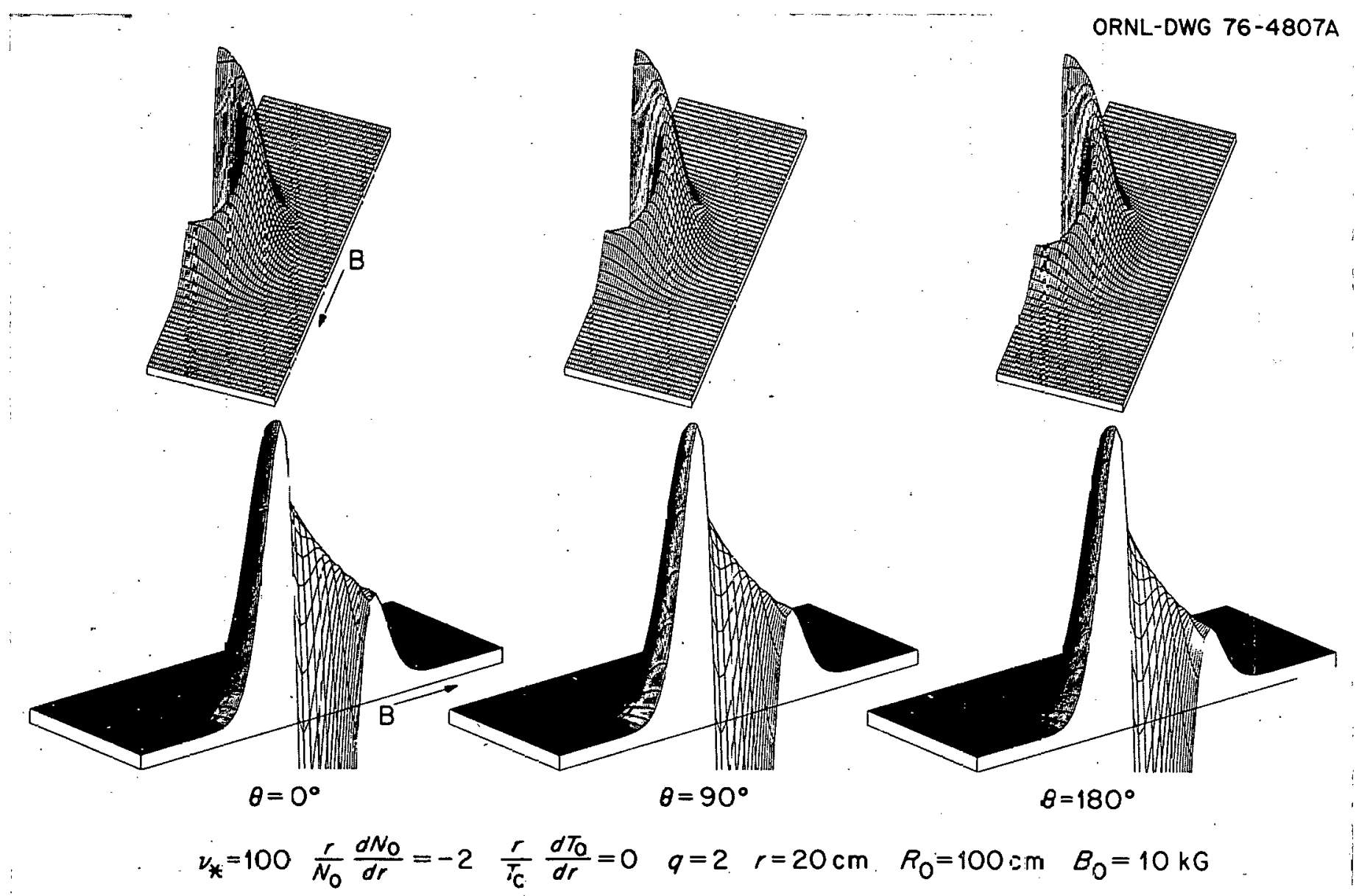

Three-Dimensional Plots of $\hat{f}\left(v_{11}, v_{1}\right)$ at Various $\theta$ for $V$ (ring voltage) $=10$ volts, $T_{0}=30 \mathrm{eV}$

Fig. 8 
Rodial Diffusion as a Function of Collisionality

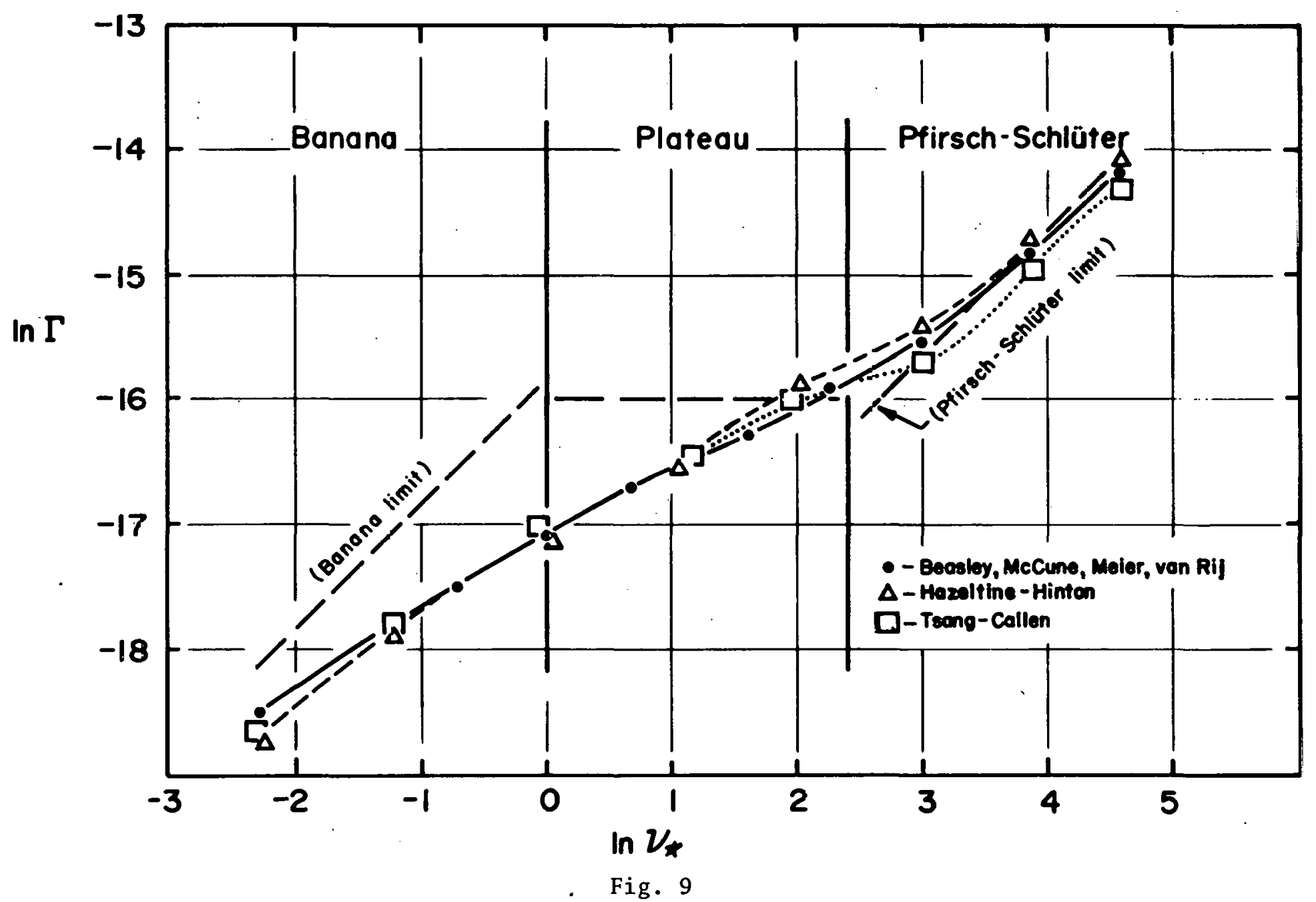


THIS PAGE

\section{WAS INTENTIONALLY \\ LEFT BLANK}


INTERNAL DISTRIBUTION

ORNL/TM-5317

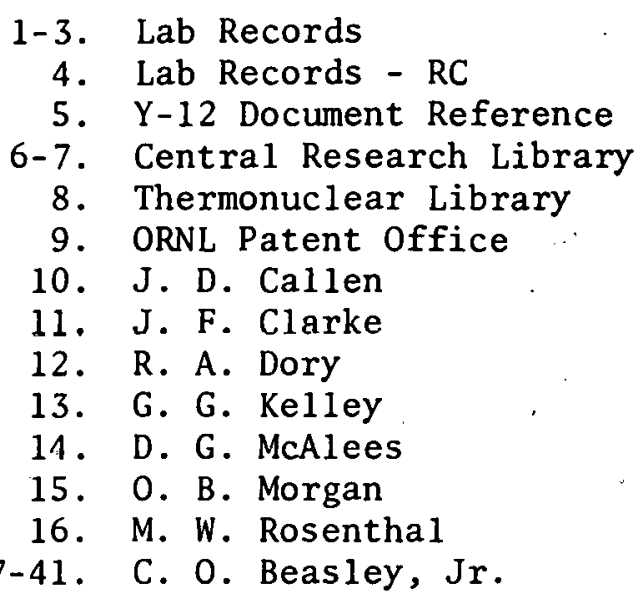

EXI'ERNAL DISTRİBUTION

42. Plasma Physics Library, Plasma Physics Laboratory, Princeton Univ., Forrestal Campus, P.0. Box 45I, Princeton, NJ 08540

43. Controlled Thermonuclear Research Library, Lawrence Livermore Laboratory, P.0. Box 808, Livermore, CA 94550

44. Q Division Library, c/o F. L. Ribe, Los Alamos Scientific Laboratory, P.0. Box.1663, Los Alamos, NM 87544

45. Controlled Thermonuclear Research Library, c/o Weston M. Stacey, Jr., Argonne National Laboratory, 9700 S. Cass Ave., Argonne, IL 60439

46. CTR Computer Center, c/o Dr. John Killeen, Lawrence Livermore Laboratory, P.0. Box 808, Livermore, CA 94550

47. Librarian, Culham Laboratory, U.K. Atomic Energy Authority, Abingdon, Oxon, OX14, 3DB, United Kingdom

48. Ruth Lengye, Bibliothek, Max Planck Institut für Plasmaphysik, 8046 Garching bei München, Federal Republic of Germany

49. Library, Centre de Récherches en Physique des Plasma, 21 Avenue des Bains, 1007, Lausanne, Switzerland

50. A. M. Dupas, Documentation S.I.G.N., Department de La Physique du Plasma, Et de La Fusion Controlée, Association EURATOM-CEA, Sur La Fusion, Centre d'Etudes Nucleaires, BP 85 Centre Du TRI 38041 Grenoble Cedex (France)

51. Bibliotheque, Service du Confinement des Plasmas, C.E.^., B.P. No. 6, 92, Fontenay-aux-Koses (Seine) France 
52. Library, International Centre for Theoretical Physics, Trieste, Italy

53. Library, Laboratorio Gas Ionizati, Frascatti, Italy

54. V. E. Ivanov, Physica1-Technical Institute of the Ukranian Academy of Sciences, Sukhumi, U.S.S.R.

55. M. S. Rabinovich, Lebedev Institute of Physics, Academy of Sciences of the U.S.S.R:, Leninsky Prospect 53, Moscow, U.S.S.R.

56. Thermonuclear Laboratory, Kurchatov Institute of Atomic Energy, 46 Ulitsa Kurchatova, P.0. Box 3402, Moscow, U.S.S.R.

57. Library, Institute for Plasma Physics, Nagoya Unịersity, Nagoya, Japan 464

58. Library, FOM-Institut voor Plasma-Fysica, Rijnhuizen, Jutphaas, Netherlands

59. Plasma Physics Group, Department of Engineering Physics, Australian National University, P.0. Box 4, Canberra A.C.T. 2600 Australia

60. Thermonuclear Library, Japan Atomic Energy Research Institute, Tokai, Naka, Ibaraki, Japan

61. CTR Reading Room, c/o Prof. Dieter J. Sigmar, Room 37-391, MİT, Cambridge, MA 02139

62. Cl'R Keading Koom, c/o Prof. D. W. Kerst, Dept. of Physics, Storling Hall, Univ. of Wisconsin, Madison, WI 53706

63. CTR Reading Room, c/o Prof. I. B. Bernstein, Yale Univ., New Haven, CT 06510

64. Center for Plasma Physics and Thermunuclear Research, c/o D. W. Ross, Univ. of Texas, Physics Dept., Austin, TX 78712

65. CTR Reading Room, c/o Prof. B. D. Fried, Physics Dept., Univ. of California, Los Angeles, CA 90024

66. CTR Reading Room, c/o Prof. David C. Montgomery, Physics \& Astronomy Dept., Univ. of Iowa, Iowa City, IA 52240

67. Magneto-Fluid-Dynamics Library, Courant Inst. of Math. Sci., New York Univ., 251 Mercer St., New YYork, NY 10012

68. CTR Reading Room, c/o Prof. Allan N. Kaufman, Physics Dept., Univ. of California, Berkeley, CA 94720 
69. CTR Reading Room, c/o Prof. W. B. Thompson, Physics Dept., Univ. of California, San Diego, La Jolla, CA 92037

70. CTR Reading Room, c/o Prof. Alvin W. Trivelpiece, Dept. of Physics \& Astronomy, Univ. of Maryland, College Park, MD 20742

71. CTR Reading Room, c/o Prof. T. Kammash, 103 Research Admin. B1dg., N. Campus, Univ. of Michigan, Ann Arbor, MI 48105

72. CTR Reading Room, c/o Dr. Ravi N. Sudan, Phillips Hall, Cornell Univ., Ithaca, NY 18450

73. Prof. Marshal1 N. Rosenbluth, Institute for Advanced Study, Princeton, $\mathrm{NJ} \cdot 08540$

74. CTR Reading Room, c/o Prof. R. Gross, Plasma Research Lab., Columbia Univ:, New York, NY 10027

75. CTR Reading Room, c/o Prof. Roy Gould, California Inst. of Tech., Pasadena, CA 91103

76. Dr. Nicholas A. Kral1, Science Applications, Inc., P.0. Box 2354, 1200 Prospect St., La Jolla, CA 92037

77. CTR Reading Room, c/o Dr. Jay P. Boris, Plasma Physics, Naval Research Laboratory, Washington, DC 20390.

78. CTR Library, General Atomic Co., P.0. Box 81608, San Diego, CA 92138

79. CTR Library, c/o Dr. Alan F. Haught, United Technologies Research Labs; East Hartford, CT $06108^{\prime}$

80. Dr. Robert E. Price, Division of Controlled Thermonuclear Research, Energy Research and Development Administration, Washington, DC 20545

81. Dr. Bennett Miller, Diviśion of Controlled Thermonuclear Research, Energy Research and Development Administration, Washington, DC 20545

82. Dr. Arthur Sleeper, Division of Controlled Thermonuclear Research, Energy Research and Development Administration, Washington, DC 20545

83. Dr. Walter Sadowski, Division of Controlled Thermonuclear Research, Energy. Research and Development Administration, Washington, DC 20545

84. Dr. D. H. Priester, Division of Controlled Thermonuclear Research, Energy Research and Development Administration, Washington, DC 20545 
85. Dr. E. E. Kintner, Division of Controlled Thermonuclear Research, Energy Research and Development Administration, Washington, DC 20545

86. Dr. L. D. Pearlstein, L-388, Lawrence Livermore Laboratory, P.0. Box 808, Livermore, CA 94550

87. Dr. J. P. Friedberg, Los Alamos Scientific Laboratory, Los Alamos, NM 87544

88. Dr. David J. Rose, Dept. of Nuclear Engineering, MIT, Cambridge, MA 02139

89. Dr. Gareth E. Guest, Gencral Atomic Co., P.0. Box 81608, Sän Dlego, CA 92138

yu. Dr. Claude Mercier, Sèrvice du l'hẻoric des Plasmas, Centre d'Etudes Nucleaires, Fontenay-aux-Roses (Seine) France

91. Dr. J. B. Taylor, Culham Laboratory, UKAEA Abingdon, Oxon, OX14 3DB, United Kingdom

92. Dr. D. Pfirsch, Institute for Plasma Physics, 8046 Garching bei München, Federal Republic of Germany

93. Dr. V. D. Shafranov, I. V. Kurchatov Inst. of Atomic Energy., 46 Ulitsa Kurchatova, P.O. Box 3402, Moscow, U.S.S.R.

94. Dr. Harold Grad, Courant Inst. of Math. Sci., New York Univ., 251 Mercer St., New York, NY 10012

95. Dr. J. G. Cordey, Culham Laboratory, UKAEA, Ab1ngdon, Oxon, Ox14, 3DB, United Kingdom

96. Dr. David Baldwin, L-388, Lawrence Livermore Lab., P.0. Box 808, Livermore, CA 94550

97. Prof. Bruno Coppi, Dept. of Physics, MIT, Cambridge, MA 02139

98. Dr. Harold P. Furth, Princeton Plasma Physics Lab., Princeton Univ,, P..0. Bux 451, Princetón, NJ .08540

99. Dr. Paul H. Rutherford, Princeton Plasma Physics Lab., Princeton Univ., P.0. Box 451, Princeton, NJ 08540

100. Dr. Herbert H. Woodson, Dept. of Electrical Engineering, Univ, of Texas, Austin, TX 78712

101. Research \& Technical Support Div., ORO, ERDA

102-128. Tech. Information Center, ERDA, OR 